\title{
On the application of Mesoscopic Eulerian Formalism to modulation of turbulence by solid phase
}

\author{
Z. Zeren ${ }^{1,2}$ \& B. Bédat ${ }^{1,2}$ \\ ${ }^{1}$ Institut de Mécanique des Fluides de Toulouse, France \\ ${ }^{2}$ CNRS, France
}

\begin{abstract}
Recently developed method, Mesoscopic Eulerian Formalism, is searched for its extension to the gas-solid flows where the carrier phase is modified by the solid phase. The possibility is shown to be existing by the introduction of two classes of particles with all the same properties except their initial positions. Classes are distributed homogeneously in space and only one of them is two-way coupled with the flow. The others are with ghost particles (particles with one-way coupling). With increased number of ghost particles, the field of source terms' of classes become similar letting the fluid realization become the same for each class. Then the conditional one-particle probability density function is definable.

Keywords: Mesoscopic Eulerian Formalism, two-way coupling, particle and fluid realization, initial particle conditions.
\end{abstract}

\section{Introduction}

Spatial distribution of inertial particles in a turbulent flow is very important in understanding different phenomena occurring in gas-solid flows such as particleparticle interactions, interactions fluid-particle, etc. For example, it is shown by Sundaram and Collins [1] that particle spatial distributions can cause significant changes in the collision rates of particles. On the other hand, particle distribution can also have specific effect on the modulation of fluid turbulence, Elgobashi and Truesdell [2].

In this regard, Fevrier et al. [3] have proposed the Mesoscopic Eulerian Formalism (MEF) to have comprehensive understanding on the distribution of 
finite inertia particles in a turbulent flow. The particle spatial velocity correlations are assumed to be induced only via the interactions with the fluid. Specifically, hydrodynamic interactions and inter-particle collisions are assumed not to induce any spatial correlation between the particles. This considers the fact that dispersed phase statistical quantities do not depend on the initial conditions of particles having chaotic motion. The statistical measures are developed then via the definition of one-particle probability density function, $\widetilde{f}_{p}^{(1)}\left(c_{p}, x, t, H_{f}\right)$, conditioned on the single fluid realization.

The extension of the method to the gas-solid flows with inter-particle collisions is proposed by Fevrier et al. [3] for dilute regimes. However, implementation in turbulence modulation regimes is not that direct due to the fact that the single fluid realization, $H_{f}$, is not obvious to be definable.

In this paper, it is shown that Mesoscopic Eulerian Formalism is applicable through the introduction of two classes of particles into a turbulent flow where only one of the classes is coupled with the flow (two-way coupling) and the other is assumed to be ghost particles (particles with one-way coupling). Particles are point sources tracked in the Lagrangian frame of reference whereas the fluid is solved in the Eulerian grid. Particle Source In Cell approximation (PSIC), initialized by Crowe et al. [4], is shown to be not to cause any significant non-physical oscillations on the fluid velocity. Discussion will continue with describing the numerical scheme and the application to two-way coupling will be discussed.

\section{Governing equations and numerical configuration}

The studied configuration is a cubical domain with a volume of $L_{b}^{3}=(2 \pi)^{3}$. The domain is discretized with $128^{3}$ grid points with periodic boundary conditions for the both phases.

Homogeneous isotropic turbulence is generated and kept stationary using a stochastic forcing scheme $[5,6]$. The code used is finite difference code with $6^{\text {th }}$ order spectral-like scheme in space and $3^{\text {rd }}$ order Runge-Kutta scheme in time. The code is parallelized with MPI (Message Passing Interface) library.

Governing equations of the fluid including the effect of particles are written as:

$$
\begin{gathered}
\frac{\partial \rho}{\partial x}+\frac{\partial \rho u_{i}}{\partial x_{i}}=0 \\
\frac{\partial \rho u_{i}}{\partial t}+\rho u_{j} \frac{\partial u_{i}}{\partial x_{j}}=-\frac{\partial p}{\partial x_{i}}+\frac{\partial \tau_{i j}}{\partial x_{j}}+\Pi_{i}+f_{i}
\end{gathered}
$$

Equations (1) and (2) are respectively for the conservation of mass and momentum.

While solving the Navier-Stokes equations in the Eulerian frame, particles are tracked individually in the Lagrangian frame. The effect of the particles on the fluid is taken into account through the term, $\Pi_{i}$, on the right hand side of the momentum equation. Without taking into account the effect of gravity, this term in the context of point-source approximation is written as: 


$$
\Pi_{i}=-\sum_{n=1}^{N_{p}} F_{p, i}^{(n)}\left(x_{p, i}^{(n)}\right) \delta\left(x-x_{p, i}^{(n)}\right)
$$

where $F_{p, i}^{(n)}$ is the force applied by the fluid to the $n^{t h}$ particle in $i$ direction defined as:

$$
F_{p, i}^{(n)}=-\frac{m_{p}^{(n)}}{\tau_{p}^{(n)}}\left(u_{p, i}^{(n)}-u_{f @ p, i}^{(n)}\right)
$$

where $u_{f @ p}$ is the fluid velocity at the position of particle and $\tau_{p}$ is the particle relaxation time defined as:

$$
\tau_{p}^{(n)}=\frac{\rho_{p} d_{p}^{2}}{18 \mu_{f} f_{D}}
$$

$f_{D}=\left(1+0.15 R e_{p}^{0.687}\right)$ is the correction to take into account the effect of particle Reynolds number, $R e_{p}$. Fluid velocity at the position of particle, $u_{f @ p}$, is calculated by $3^{\text {rd }}$ order Lagrangian interpolation scheme.

The term $f_{i}$ in the equation 2 is the stochastic forcing term which keeps the turbulence stationary.

\section{Initial conditions of particle phase}

Characteristics of turbulence and particles can be found in table 1 and table 2 . $T_{E}$ is the Eulerian time scale calculated by the Eulerian one-point autocorrelation function, $T_{e}$ is eddy turnover time defined as $L_{f} / u^{\prime}$ where $L_{f}$ is the longitudinal large scales length and $u^{\prime}$ is the characteristic velocity of turbulence. $L_{g}$ is the transversal large scales. $T_{L_{f}}$ is Lagrangian time scale of large structures calculated by the Lagrangian one-point autocorrelation function. Based on these values, turbulent Reynolds number is defined as $R e_{L}=u^{\prime} L_{f} / \nu_{f}$ and Reynolds number based on the Taylor scales is defined as $R e_{\lambda}=u^{\prime} \lambda_{g} / \nu_{f}$.

The crucial condition for MEF to be applicable is that the particle spatial correlations are induced only by the interaction with the fluid. As mentioned in introduction, this dictates the fact that the hydrodynamic interactions and interparticle collisions are assumed not to induce any spatial correlation. To this end, small deviations in the initial conditions are quickly magnified, after several relaxation time, particle statistics become independent of the initial conditions and controlled by the interactions with the fluid.

Under these assumptions, two populations of particles are introduced into the same fluid realization with the same mesoscopic fields except their positions. The characteristics of particles and of turbulence are defined in table 1 and table 2. Note that in the table, total number of particles is given. Each populations is then with 2 particles per grid in $D N S 128^{3}$ application. These two populations are timestepped with the fluid realization without two-way coupling so that they settle to an equilibrium with the turbulent field. Their statistical quantities, particle kinetic 
Table 1: Turbulence characteristics without two-way coupling.

\begin{tabular}{cccccccc}
\hline$T_{E}$ & $T_{E} / T_{e}$ & $T_{L_{f}} / T_{E}$ & $L_{f} / L_{b}$ & $L_{f} / L_{g}$ & $\eta_{f}$ & $R e_{L}$ & $R e_{\lambda}$ \\
\hline 6.2816 & 1.0267 & 0.8275 & 0.0864 & 1.9797 & 0.0177 & 96 & 49 \\
\hline
\end{tabular}

Table 2: Particle characteristics.

\begin{tabular}{ccc}
\hline$\rho_{p} / \rho_{f}$ & $d_{p} / \eta_{f}$ & $N_{p_{\text {total }}}$ \\
\hline 12000 & 0.0751 & $4 \times 128^{3}$ \\
\hline
\end{tabular}

Table 3: Fluid and particle statistics.

\begin{tabular}{lllll}
\hline Population & $q_{p}^{2}$ & $q_{f}^{2}$ & $q_{f p}$ & $q_{f @ p}^{2}$ \\
\hline Class $A$ & $.4544 \mathrm{E}-02$ & $.1176 \mathrm{E}-01$ & $.8883 \mathrm{E}-02$ & $.1141 \mathrm{E}-01$ \\
Class $B$ & $.4546 \mathrm{E}-02$ & $.1176 \mathrm{E}-01$ & $.8883 \mathrm{E}-02$ & $.1141 \mathrm{E}-01$ \\
\hline
\end{tabular}

energy $q_{p}^{2}$, turbulent kinetic energy $q_{f}^{2}$, fluid-particle covariance $q_{f p}$, turbulent kinetic energy seen by the particles $q_{f @ p}^{2}$, in stationarity are shown in table 3 and they're exactly the same. This final field obtained is referred as $T^{+} / \tau_{p}=0$ where $T^{+}$is the nondimensional time of the simulations. These populations will be useful not only for the verification of the PSIC approximation but also for the test that is performed to validate the MEF's application to two-way coupling.

Initial distributions of the particles are shown in fig. 1. It is clear that the both classes are distribution homogeneously in space.

To be more quantitative on the distributions of the both classes, the normalized spatial distribution function, $P(C)$, which is possible number of particles, $C$, in an elementary volume and longitudinal spatial correlations of particles, $R_{p p}(r)=<$ $u_{p}(x) u_{p}(x+r)>$, are plotted. Shown in fig. 2 , both populations have exactly the same correlation and distribution curves validating the equality of the mesoscopic fields of both classes.

\section{Validity of point source approximation}

Once being sure of the initial conditions of the particle classes, a numerical study has been performed to verify the point source approximation (PSIC). It is been shown by Eaton [7] how the point source approximation fails with increase in the particle radius. In this paper, particles smaller than the Kolmogorov scale are used (see table 2) so the effect of wake production is negligible. Then all the rest is the validation of the approximation. 

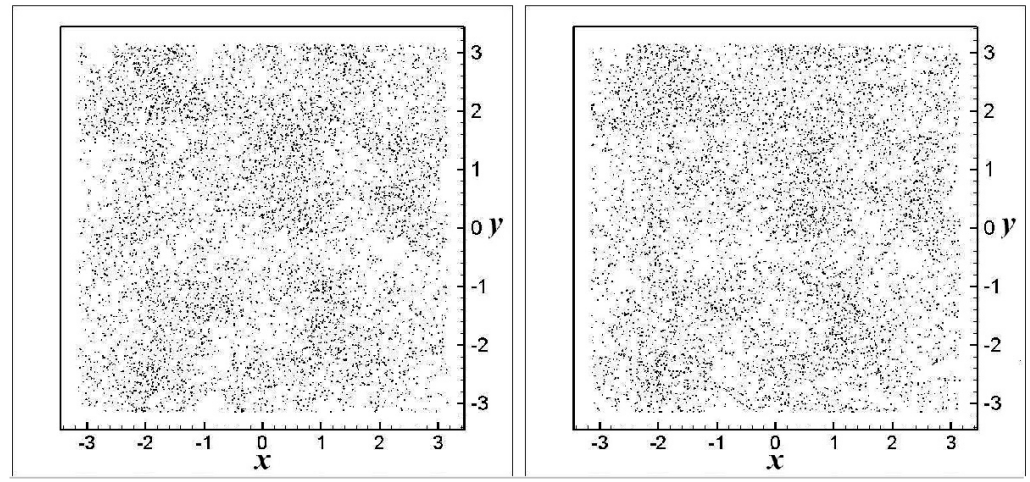

Figure 1: Spatial distribution of particles, $T^{+} / \tau_{p}=0$. Class $A$ on the left and Class $B$ on the right.
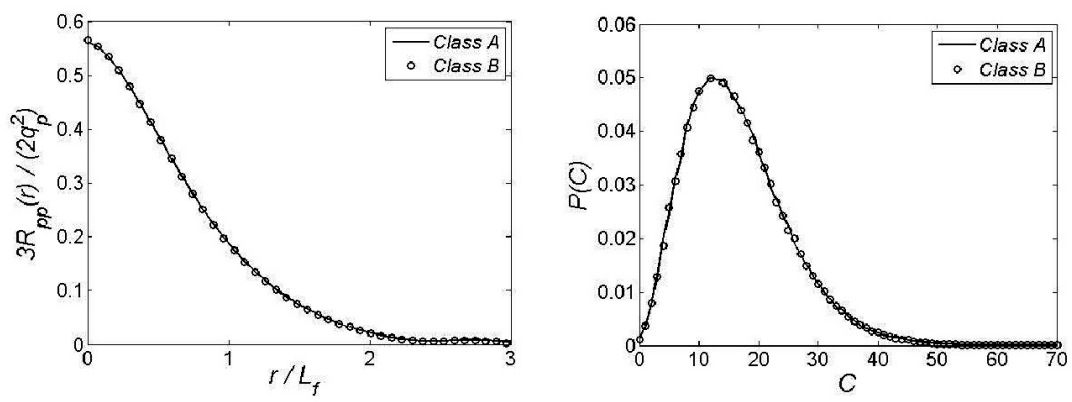

Figure 2: Spatial correlations, $R_{p p}(r)$ normalized by particle phase kinetic energy, $q_{p}^{2}$, and distribution functions of particle classes, $P(C)$, at $T^{+} / \tau_{p}=0$.

Simple test is then applied to two-classes of particles introduced into the same fluid realization. One of them is coupled with the fluid and the other is chosen as the ghost particles (particles with one-way coupling) (see Vermorel et al. [8]). If the approximation is to be valid, then the statistical values of each class should be similar with a negligible difference between each other so that they see the same fluid field.

As seen in fig. 3, the difference of the fluid-particle covariance, $q_{f p}$, between the two populations is less than $1 \%$ which is rather acceptable for the application.

\section{Application of MEF to two-way coupling}

As explained in introduction, for the MEF's application to the flows where the two-way coupling cannot be ignored, the definition of the probability density 


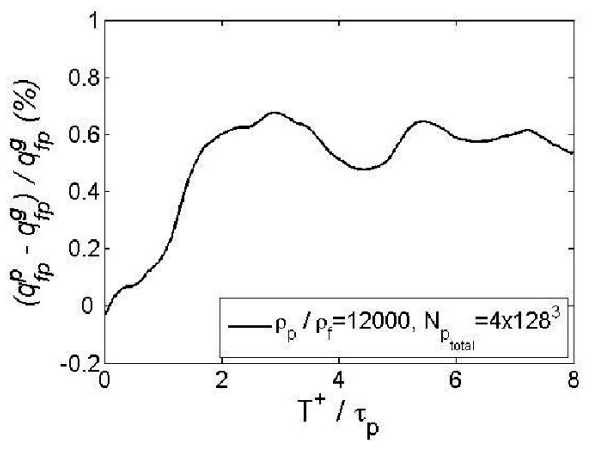

Figure 3: The error of the PSIC approximation, $q_{f p}^{p}$ is particle-fluid covariance for the particles effecting the flow and $q_{f p}^{g}$ is covariance for the ghost particles.

function, $\tilde{f}_{p}^{(1)}\left(c_{p}, x, t\right)$, is not direct forward preventing the definitions of statistical quantities conditioned on a single realization of fluid turbulence.

Initially, the idea was to explore the effect of different initial conditions of particles on the same fluid field, $H_{f}$. Performing number of two-way coupled simulations with particles having different initial positions, it was observed how the turbulence evolves with a response to different initial conditions. Specifically, it was curious to find a time range where the fluid turbulence does not differ between different fluid realizations enormously. To compare the fluid fields in these different simulations, the proper method is the utilization of the normalized parameter $D$ :

$$
D=\left(<\left(u_{A}-u_{B}\right)^{2}>/ u_{A_{r m s}} u_{B_{r m s}}\right)^{1 / 2}
$$

where $u_{A}$ is the fluid velocity with response to the Class $A$ and $u_{B}$ is the one to Class $B$ where $<$. $>$ denotes volume averaging.

Two simulations are performed with 2 classes of particles (see table 2). The particle initial conditions are as explained in section 3. Fig. 4 shows the behavior of $D$ and the particle statistics in time. As seen in the figure, the flow fields differentiate more than $10 \%$ and significantly the difference increase in time whereas the statistical quantities are the same for each class (the figure on the right hand side).

This difference in instantaneous fluid field is due to the small deviations in the feedback of particles which are quickly amplified in time by the non-linear chaotic nature of turbulence. The conclusion is then the turbulent field does not allow a fluid realization rest the same at least for a small time-range when it is coupled with the particle phase.

To come over the non-linear nature of turbulence, the simulation resumed in the first test is considered with only one class being active (two-way coupled to the fluid) and the other class is considered as ghost particles (particles with 

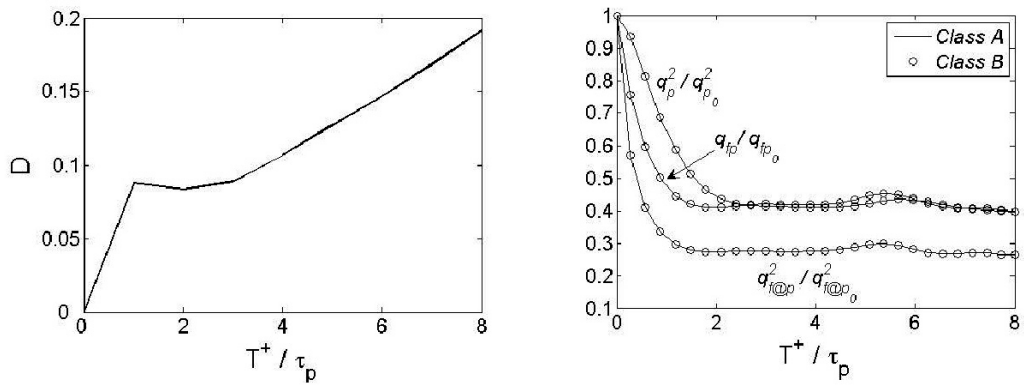

Figure 4: Parameter $D$, the difference between the fluid realizations and statistics of the two classes, Class $A$ and ClassB.
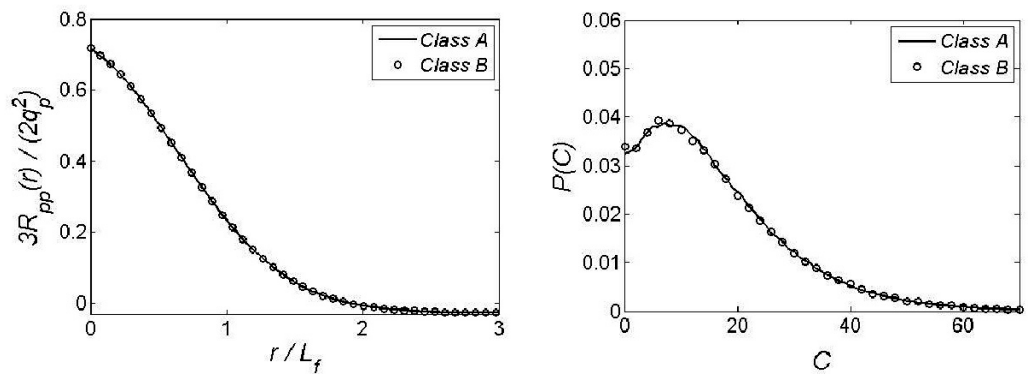

Figure 5: Spatial correlations, $R_{p p}(r)$ normalized by particle phase kinetic energy, $q_{p}^{2}$, and distribution functions of particle classes, $P(C)$, at $T^{+} / \tau_{p}=8$.

one-way coupling). In the same fluid realization, difference between the source terms of classes are then expected to be dependent on the number of particles, at least for a time range. Two simulations are performed with the configuration where $C l a s s A$ and $C l a s s B$ are the ones active in respective simulations to see the interchangability of the active class. As might be guessed, different classes correspond to different initial conditions and the fundamentality here is then the ghost particles' utility which is to increase the precision of the computation of the mesoscopic quantities using the p.d.f. $\widetilde{f}_{p}^{(1)}$.

As seen in fig. 5, at the end of the simulation, spatial distributions and correlations of the two classes rest the same to each other, shown only for one simulation. This is to say that even with two-way coupling, particle field keeps the mesoscopic field, as in the initial conditions, the same for both classes.

However, the effect on the fluid field is not the same when the active class is $A$ or $B$. Turbulent field responding to Class $A$ and $C l a s s B$ in respective simulations is shown in fig. 6. It is clear that the topology of the flow stays the same. However, analyzing closer the field, the dashed-line regions are shown in fig. 7. The dots in 

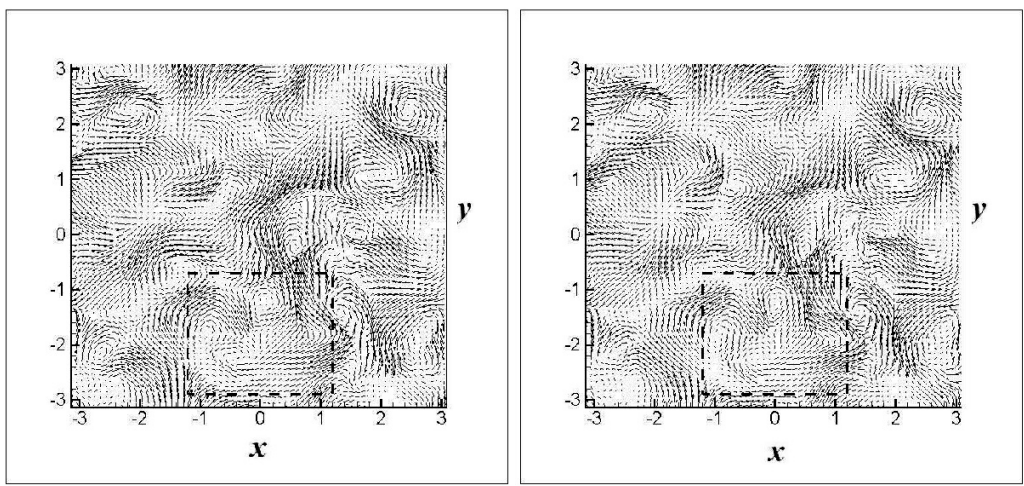

Figure 6: Fluid velocity vectors, $T^{+} / \tau_{p}=8$, on the left hand side, Class $A$ is active, on the right hand side, Class $B$ is active in seperate realizations.
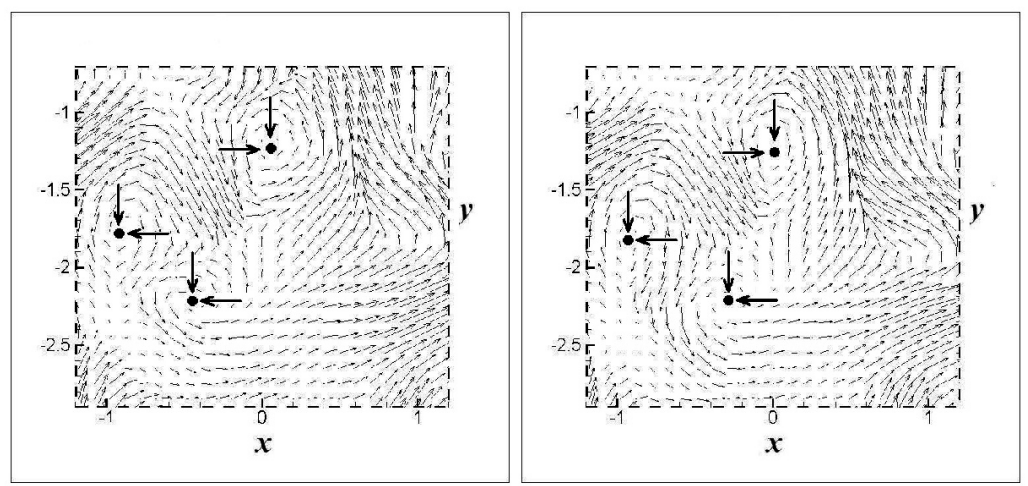

Figure 7: Fluid velocity vector zoom field, the dashed-line regions in fig. 6.

the fields shows the approximative centers of the vortexes and as seen, they have slight deviations between each other.

The source terms of active and non-active classes in one of the simulations are shown in fig. 8. As seen in the figure, the difference quantified by the parameter $\mathrm{D}$ in time stays constant for a time range more than one particle relaxation time. The initial peak on the graph is due to the transition of both the particles and fluid field to arrive at a new equilibrium, it is to be reminded that turbulence is forced by the scheme of Eswaran and Pope [5]. In the stationary period, constant difference seems like promising and more strictly, it has been found out that the difference depends on the number of ghost particles, not shown here. As the number of particles in each class, one-way or two-way, increases the parameter $D$ decreases.

So there is a statistical relation between the number of particles and the difference of the source terms among the classes. This sounds a bit like the statistical 

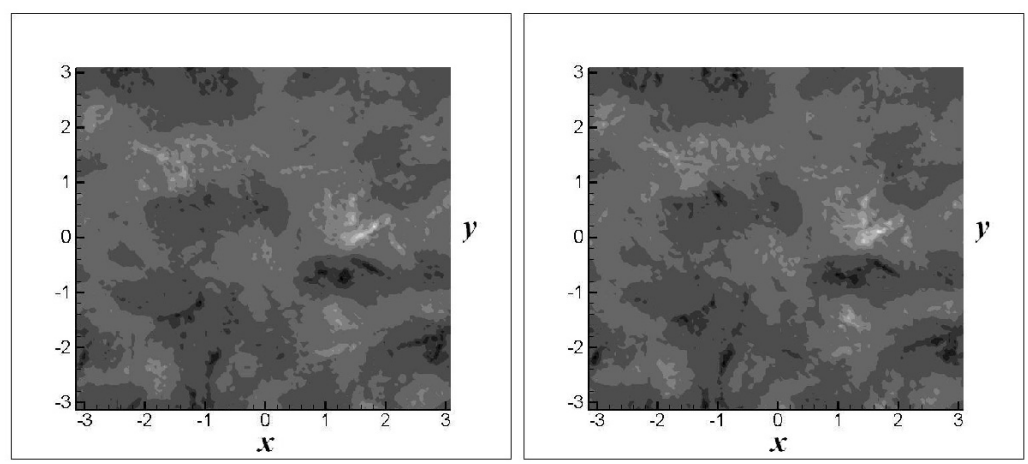

Figure 8: Source term $\Pi_{x}$, eq. (3), of particles in $x$ direction at $T^{+} / \tau_{p}=8$, of Class $A$ (active) on the left and of ClassB (non-active) on the right in the same fluid realization.

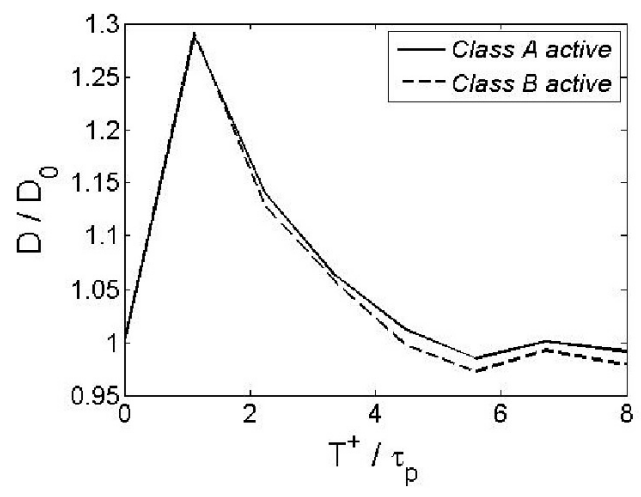

Figure 9: Parameter $D$, difference between the source terms of particles in Class $A$ and Class $B$ for the two realizations of fluid.

confirmation of PSIC method. Using high number of particles lets the changing the active class to any other classes to generate the same fluid field. The definition of the conditional probability density function, $\widetilde{f}_{p}^{(1)}\left(c_{p}, x, t, H_{f}\right)$, is then possible.

\section{Conclusion and perspectives}

The Mesoscopic Eulerian Formalism is shown to be applicable to the flows where the carrier phase is modified by the presence of solid particle phase. From statistical point of view, increasing the number of particles reduces the error of the PSIC approximation where non-physical oscillations are not obtained and also high number of particles lets the definition of a single fluid realization, $H_{f}$, in which large number of particle realizations, $H_{p}$, is imaginable. Study can 
be extended to different Stokes numbers to see the difference when there is no concentration of particles. Also classes with different numbers of particles should be very informative on the effects of initial conditions. With the definition of the probability density function of particles conditioned on a single fluid realization, mesoscopic field values become measurable.

\section{Acknowledgements}

This research project has been supported by a Marie Curie Early Stage Research Training Fellowship of the European Community Sixth Framework Program under contract number MEST-CT-2005-020426'.

\section{References}

[1] Sundaram, S. \& Collins, L.R., Collision statistics in an isotropic particle-laden turbulent suspension. part 1. direct numerical simulations. Journal of Fluid Mechanics, 335, pp. 75-109, 1997.

[2] Elghobashi, S. \& Truesdell, G.C., On the two-way interaction between homogeneous turbulence and dispersed solid particles. part 1: Turbulence modification. Phys Fluids A, 5(7), pp. 1790-1801, 1993.

[3] Fevrier, P., Simonin, O. \& Squires, K.D., Partitioning of particle velocities in gas-solid turbulent flows into a continuous field and a spatially uncorrelated random distribution: theoretical formalism and numerical study. Journal of Fluid Mechanics, 533, pp. 1-46, 2005.

[4] Crowe, C., Sharm, M. \& Stock, D., The particle source in cell (psi-cell) model for gas-droplet flows. J Fluids Engineering, 99, pp. 325-332, 1977.

[5] Eswaran, V. \& Pope, S., An examination of forcing in direct numerical simulations of turbulence. Computers and Fluids, 16, pp. 257-278, 1988.

[6] Zeren, Z. \& Bédat, B., Spectral and physical forcing of turbulence. Proceedings of the iTi International Conference on Turbulence, Bertinoro, Italy, 2008.

[7] Eaton, J.K., Two-way coupled turbulence simulations of gas-particle flows using point particle tracking. International Journal of Multiphase Flow, In Press, Accepted Manuscript.

[8] Vermorel, O., Bédat, B., Simonin, O. \& Poinsot, T., Numerical study and modelling of turbulence modulation in a particle laden slab flow. Journal of Turbulence, 4, p. 25, 2003. 